\title{
Void-containing materials with tailored Poisson's ratio
}

\author{
Olga A. Goussev ${ }^{a)}$ and Peter Richner \\ Swiss Federal Laboratory for Materials Testing and Research, Üeberlandstrasse 129, \\ CH-8600 Düebendorf, Switzerland \\ Michael G. Rozman and Andrei A. Gusev \\ Institute of Polymers, Department of Materials, ETH-Zentrum, CH-8092 Zürich, Switzerland
}

(Received 7 February 2000; accepted for publication 22 June 2000)

\begin{abstract}
Assuming square, hexagonal, and random packed arrays of nonoverlapping identical parallel cylindrical voids dispersed in an aluminum matrix, we have calculated numerically the concentration dependence of the transverse Poisson's ratios. It was shown that the transverse Poisson's ratio of the hexagonal and random packed arrays approached 1 upon increasing the concentration of voids while the ratio of the square packed array along the principal continuation directions approached 0 . Experimental measurements were carried out on rectangular aluminum bricks with identical cylindrical holes drilled in square and hexagonal packed arrays. Experimental results were in good agreement with numerical predictions. We then demonstrated, based on the numerical and experimental results, that by varying the spatial arrangement of the holes and their volume fraction, one can design and manufacture voided materials with a tailored Poisson's ratio between 0 and 1 . In practice, those with a high Poisson's ratio, i.e., close to 1 , can be used to amplify the lateral responses of the structures while those with a low one, i.e., close to 0 , can largely attenuate the lateral responses and can therefore be used in situations where stringent lateral stability is needed. (C) 2000 American Institute of Physics. [S0021-8979(00)04519-9]
\end{abstract}

\section{INTRODUCTION}

Interest in the elastic properties of voided materials in general, and their Poisson's ratios in particular, has been steady during the last decade. Thorpe and Jasiuk ${ }^{1}$ have reviewed a number of exact relationships in two-dimensional elasticity, together with some interesting applications. Cherkaev, Lurie, and Milton ${ }^{2}$ found remarkable invariance of the stress fields in two-dimensional elastic systems loaded at the boundary by fixed forces when the compliance tensor was shifted uniformly. Based on this invariance they proved that the effective Young's modulus of a two-dimensional medium with holes does not depend on the Poisson's ratio of the matrix material. Day et al. ${ }^{3}$ came to the same conclusion using computer simulations, where a continuum medium was modeled as a pixel lattice connected in a spring network under periodic boundary conditions. Three different arrangements of circular holes were studied: the honeycomb lattice, the triangular lattice, and a random one. Eischen and Torquato 4 applied a boundary element method to a hexagonal array of infinitely long, aligned cylinders embedded in a matrix. Using properties for each phase representative of commonly used materials, they tabulated the overall elastic moduli over a wide range of matrix volume fractions. For square cells with walls of constant thickness, it was demonstrated $^{5}$ that their stiffness was highly anisotropic, with two stiff and two compliant directions.

In this article we study, both numerically and experimentally, voided materials with parallel nonoverlapping identical cylindrical holes drilled in various spatial arrangements and hole volume fractions, taking as a particular example an aluminum matrix (Fig. 1).

${ }^{a)}$ Electronic mail: olga.goussev@empa.ch
Our goal is to identify voided materials with tailored transverse Poisson's ratios. It is worth noting that for anisotropic materials, the upper and lower bounds for Poisson's ratio are 1 and -1 , respectively (for isotropic materials the bounds are 0.5 and -1$){ }^{6}$ In practice, voided materials with a high Poisson's ratio, i.e., close to 1 , can be used to amplify the lateral responses of the structures while those with a low value close to 0 , can largely attenuate the lateral responses and can therefore be used in situations where a stringent requirement on the lateral stability is imposed.

\section{NUMERICAL}

We studied numerically the effective elastic properties of voided materials in three different arrangements of nonoverlapping circular holes: hexagonal, square, and random (Fig. 2).

Periodic morphology-adaptive meshes comprised of up to a million triangles were used in our calculations. As an example, the mesh employed for a random structure of nine nonoverlapping identical holes is sketched in Fig. 3.

To form three-dimensional periodic models, each mesh triangle was viewed as a base of a prism with equal heights attached to each node perpendicular to the plane. An iterative conjugate-gradient solver with a diagonal Jacobi's preconditioning was employed. Three-dimensional constitutive laws were used to calculate the overall elastic constants, without assuming either plane stress or plane strain. More details on the generation of periodic morphology-adaptive meshes and the calculation of effective elastic properties can be found in Refs. 7 and 8 .

As a test, we repeated calculations assuming different Poisson's ratios for the matrix. Interestingly, the effective Young's modulus did not depend on the Poisson's ratio of 


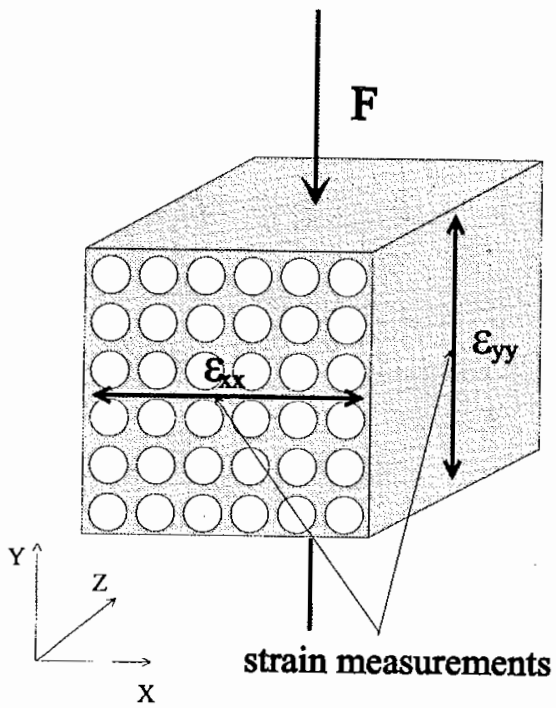

FIG. 1. Experimental arrangement studied.

the matrix material, as it should according to the predictions of Refs. 1-3.

It was found that the effective transverse Young's modulus of all structures studied decreased gradually to zero at the

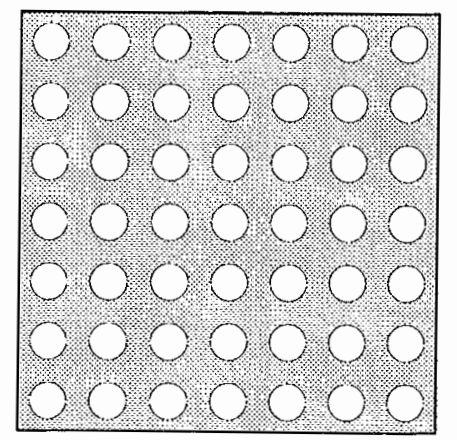

(a)

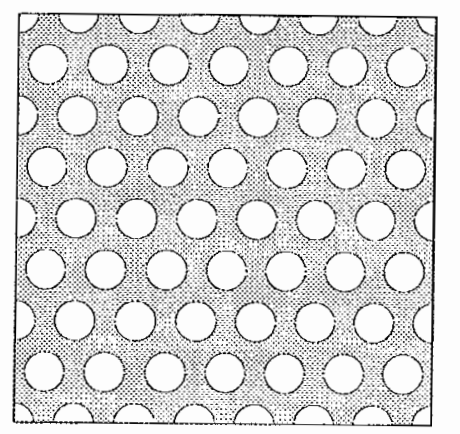

(b)

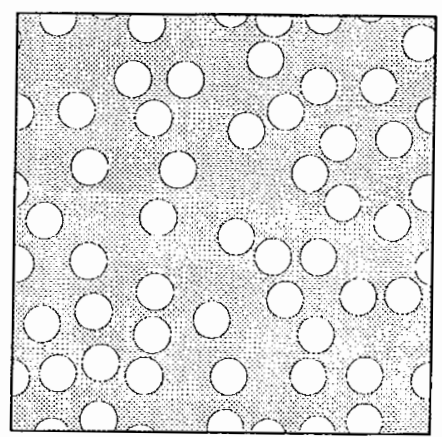

(c)

FIG. 2. Morphologies studied: (a) square; (b) hexagonal; (c) random.

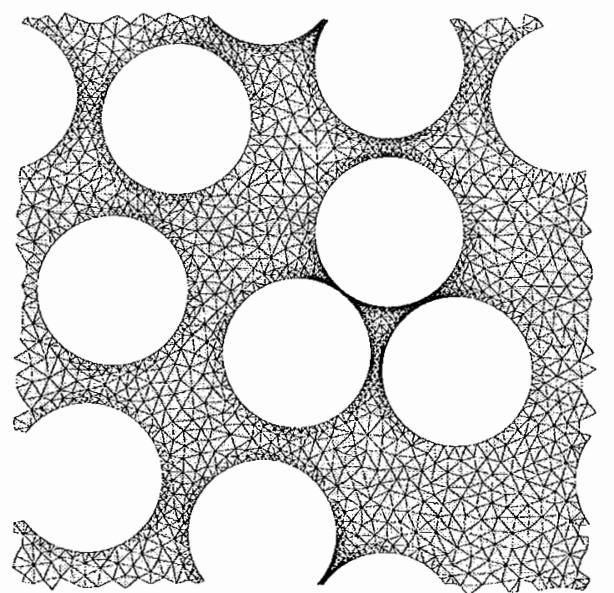

FIG. 3. A periodic morphology-adaptive mesh for a random structure.

percolation limit as the void fraction increased, with the square structure being the stiffest (when loaded in the $x$ or $y$ direction in Fig. 1) and the random the weakest [Fig. 4(a)].

Calculated values for the hexagonal array almost coincided with those calculated by Eischen and Torquato ${ }^{4}$ using a boundary element method. The deviation was typically less than 5\%. Comparison of our numerical results [Fig. 4(a)] with those of Day et $a l^{3}$ shows that for the hexagonal array the results are quite comparable (our calculations yield up to $12 \%$ stiffer structures). However, for the random structure the difference is larger. It could be explained by the fact that our model is a nonoverlapping model, whereas the model of

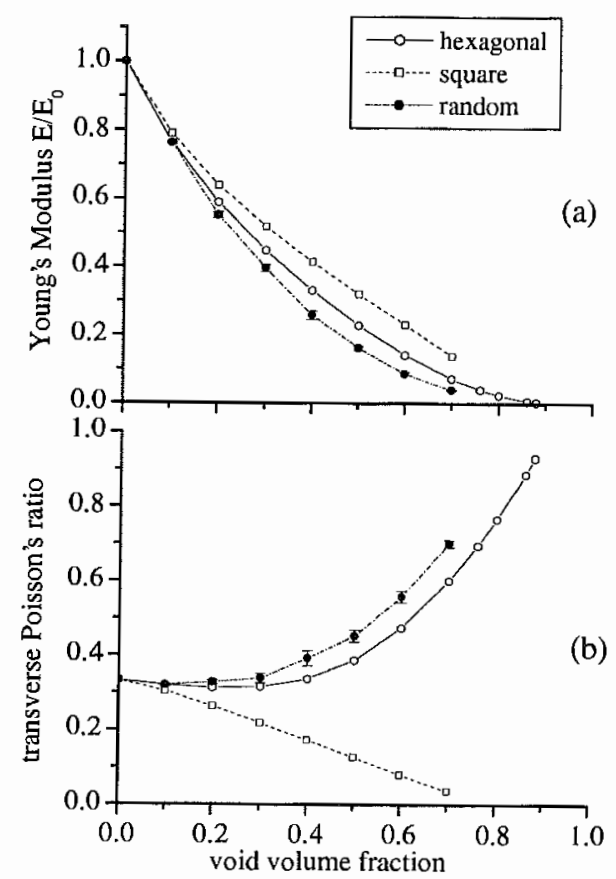

FIG. 4. Calculated effective Young's moduli (a) and Poisson's ratios (b) for the hexagonal, square, and random packed arrays. The symbol specification is given in the figure. For the random array, five independent computer configurations were generated at each void fraction studied. Calculated Young's moduli and Poisson's ratios of the individual configurations were used to evaluate the average values and the error bars shown in the figure. For the regular hexagonal and square arrays, the calculations delivered a single estimate for a given void fraction. 


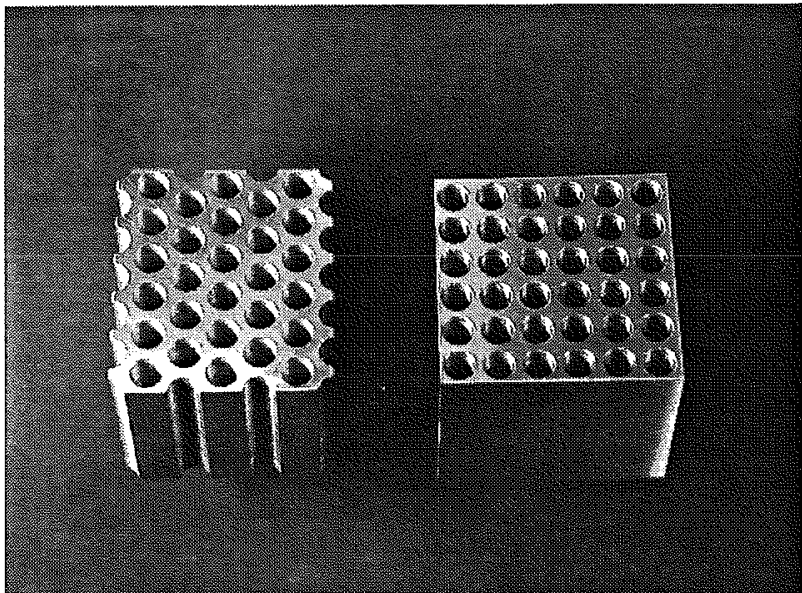

FIG. 5. Bricks of aluminum with $50 \%$ voids.

Day et al. allows overlaps between the voids, leading to a more rapid decrease of the effective Young's modulus upon increasing the void fraction.

Figure 4(b) shows that the transverse Poisson's ratios of the hexagonal and random packed arrays approached 1 upon increasing the concentration of the voids while that of the square packed array approached 0 (when loaded in the $x$ or $y$ direction in Fig. 1). For the hexagonal structure, our results for the effective Poisson's ratios faithfully reproduced those calculated with both the boundary element method ${ }^{4}$ and the discretized-spring network model, ${ }^{3}$ with a deviation of less than $11 \%$. Calculated values of the Poisson's ratio of the random structures studied differed significantly from those of Day et al. ${ }^{3}$ To the best of our knowledge, there exist no results for the transverse Poisson's ratio of the voided materials with a square arrangement of the voids.

Both the boundary element formulation employed by Eischen and Torquato ${ }^{4}$ and the finite element formulation used in our work are accurate in principle. One should therefore expect identical predictions for comparable situations. In practice, however, there are numerical imperfections (finite meshes, round-off errors, etc.) so the two methods may deliver somewhat different results. The consistent agreement between the two numerical predictions obtained for the hexagonal array validates convincingly the two implementations and makes us confident in the significance of the numerical predictions obtained in the current study. In contrast, the discretized spring-network formulation presented by Day et $a l^{3}$ involves some approximations so the moderate deviations seen between their and our predictions should perhaps be unavoidable.

Day et ll $^{3}$ studied the behavior of the hexagonal, triangular, and random arrays near the critical void fractions. They demonstrated that the behavior could be rationalized in terms of various neck formations. Here we have found that the concentration dependence of Poisson's ratio of the square array differs significantly from those typical of the random and hexagonal arrays [see Fig. 4(b)]. For example, at a void fraction of 0.4 , the square array has a Poisson's ratio of 0.17 while the random and hexagonal arrays still exhibit Poisson's ratios practically indistinguishable from that assumed

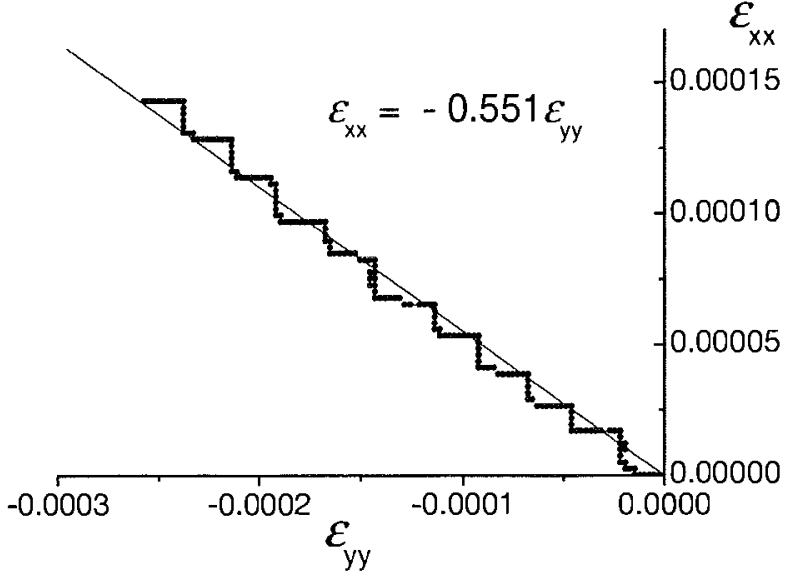

FIG. 6. A strain measurement for the hexagonal structure with $70 \%$ voids.

for the pure matrix, i.e., 0.33. At such moderate volume fraction, the size of the "necks"' between the holes in the square array is close to the radius of the holes, thus allowing one to surmize that the concentration dependence of Poisson's ratio of the square array should have a nonstructural, solid-like origin.

\section{EXPERIMENT}

Experimental measurements were carried out by measuring the Poisson's ratios of aluminum rectangular bricks under compression with 36 identical cylindrical holes drilled

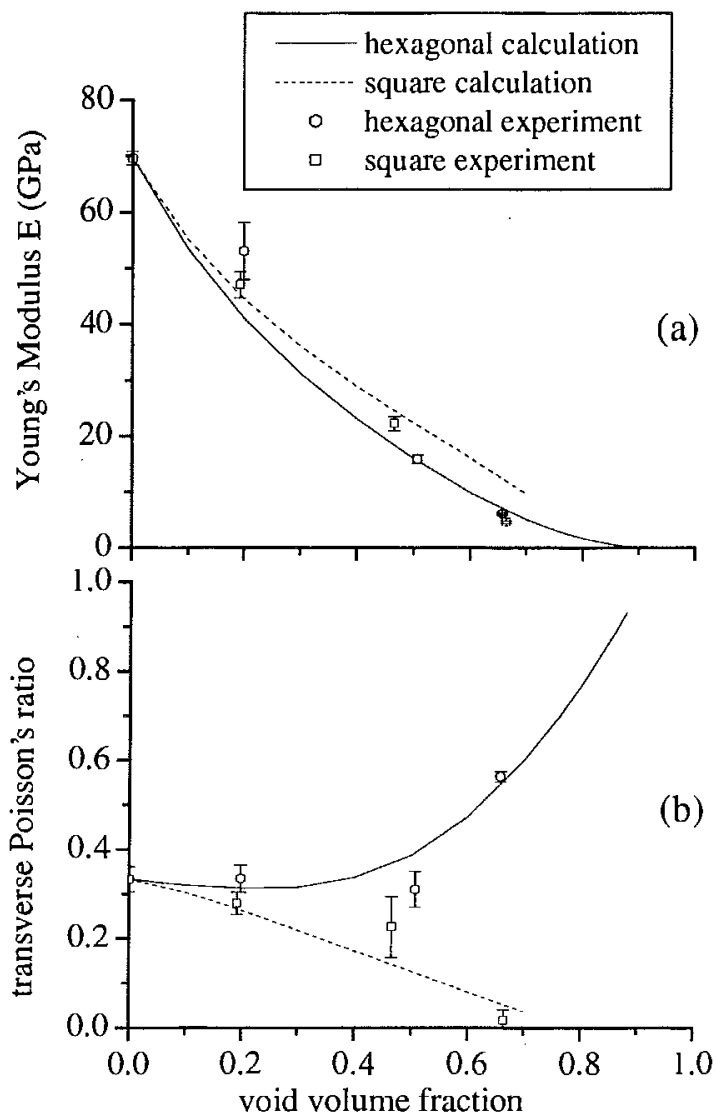

FIG. 7. Young's moduli and transverse Poisson's ratios measured on bricks of aluminum with holes. 
with both square and hexagonal packed arrays. Samples with a void volume fraction of approx. $0.2,0.5$, and 0.7 were manufactured and tested (Fig. 5).

Simultaneous independent strain measurements in both longitudinal and lateral directions were conducted on a tensile machine using additional extensometers. The average of two simultaneously measured values on the two sides of the brick was reported. The measurements were conducted in the elastic domain, with a maximal strain below $0.05 \%$. A typical strain measurement is presented in Fig. 6.

The experimental results and their comparison with numerical predictions for the Young's modulus and transverse Poisson's ratio are shown in Fig. 7.

Experimental results were in good agreement with the numerical predictions, thus allowing the use of the latter for numerical identification of voided materials with a tailored Poisson's ratio. Accounting for the fact that accurate experimental measurements of Poisson's ratio are rather tedious and time consuming, we believe that numerical techniques, such as the one used in this work, can be efficiently employed in the design of voided materials with tailored elastic properties.

\section{CONCLUSIONS}

It was demonstrated, both numerically and experimentally, that the transverse Poisson's ratio of voided materials depends strongly upon the spatial arrangement of the voids and their volume fraction. In contrary, the effective stiffness does not depend much on the void arrangement. As a result, varying the spatial arrangement of cylindrical holes and their volume fraction, one can design and manufacture voided materials with tailored Poisson's ratio between 0 and 1 . This can find different important engineering applications. For example, materials with a high Poisson's ratio, i.e., close to 1 , can be used as strain switchers redirecting the deformation applied to a direction of interest. Those with a low Poisson's ratio, close to 0 , can almost completely suppress the lateral responses and can therefore be used in situations where a stringent lateral stability is important.

\section{ACKNOWLEDGMENT}

The authors thank P. Bertschenger for help in the measurements.

${ }^{1}$ M. F. Thorpe and I. Jasiuk, Proc. R. Soc. London, Ser. A 438, 531 (1992).

${ }^{2}$ A. Cherkaev, K. Lurie, and G. W. Milton, Proc. R. Soc. London, Ser. A 438, 519 (1992).

${ }^{3}$ A. R. Day, K. A. Snyder, E. J. Garboczi, and M. F. Thorpe, J. Mech. Phys. Solids 40, 1031 (1992).

${ }^{4}$ J. W. Eischen and S. Torquato, J. Appl. Phys. 74, 159 (1993).

${ }^{5}$ L. J. Gibson and M. F. Ashby, Cellular Solids: Structure and Properties (Cambridge University Press, Cambridge, 1997).

${ }^{6} \mathrm{R}$. M. Christensen, Mechanics of Composite Materials (Wiley, New York, 1979).

${ }^{7}$ A. A. Gusev and M. G. Rozman, Comput. Theor. Polym. Sci. 9, 335 (1999).

${ }^{8}$ A. A. Gusev, P. Hine, and I. Ward, Compos. Sci. Technol. 60, 535 (2000). 